\title{
Information floating on a road with different traffic volumes between opposite lanes
}

\author{
Keisuke Nakano ${ }^{1, *}$ and Kazuyuki Miyakita ${ }^{2}$ \\ ${ }^{1}$ Department of Information Engineering, Niigata University, Niigata, 950-2181 Japan \\ ${ }^{2}$ Center for Academic Information Service, Niigata University, Niigata, 950-2181 Japan \\ *nakano@ie.niigata-u.ac.jp
}

Received: August 19, 2015; Accepted: January 12, 2016; Published: August 8, 2016

\begin{abstract}
Information floating (IF) is an invisible infrastructure in which mobile nodes can receive information that seems to be floating in a designated area. IF is realized by successively transferring information from a mobile node to other nodes in an epidemic manner with wireless direct communication in designated areas. IF has many potential applications, such as advertisements for local communities, specific communication for disasters, alert systems for pedestrians and vehicles, and so on. To design alert systems on a road, some articles theoretically analyze the mean lifetime of the floating in a one-dimensional street. In past researches, however, identical traffic volume and velocity were assumed in all the lanes. The difference in traffic volume between lanes and the correlation between traffic volume and velocity were omitted for simplicity. In this paper, we recognize the importance of these two factors and reveal their effects on the mean lifetime of IF with a new theoretical method. In it, we consider not only the effects of the above two factors but also the complicated features of IF that were ignored in previous methods.
\end{abstract}

Keywords: information floating, lifetime, opposite lanes, traffic volume, theoretical analysis

\section{Introduction}

We can currently use such node-to-node direct wireless communication as the ad-hoc modes of wireless LAN systems and Bluetooth devices as well as node-to-infrastructure wireless communication systems like cellular mobile systems and wireless LAN systems. A wireless multi-hop path, which is utilized in mobile ad-hoc networks (MANETs) [1], wireless sensor networks (WSNs) [2], and wireless mesh networks [3], is an important application for direct wireless communication and is realized by combining direct wireless communication and relaying by mobile nodes. A mobile multi-hop network can be extended to more flexible node-to-node communication in delay tolerant networks (DTN) [4, 5]. In DTNs, a connected path between end-nodes is not always needed for communication, and information is spread 


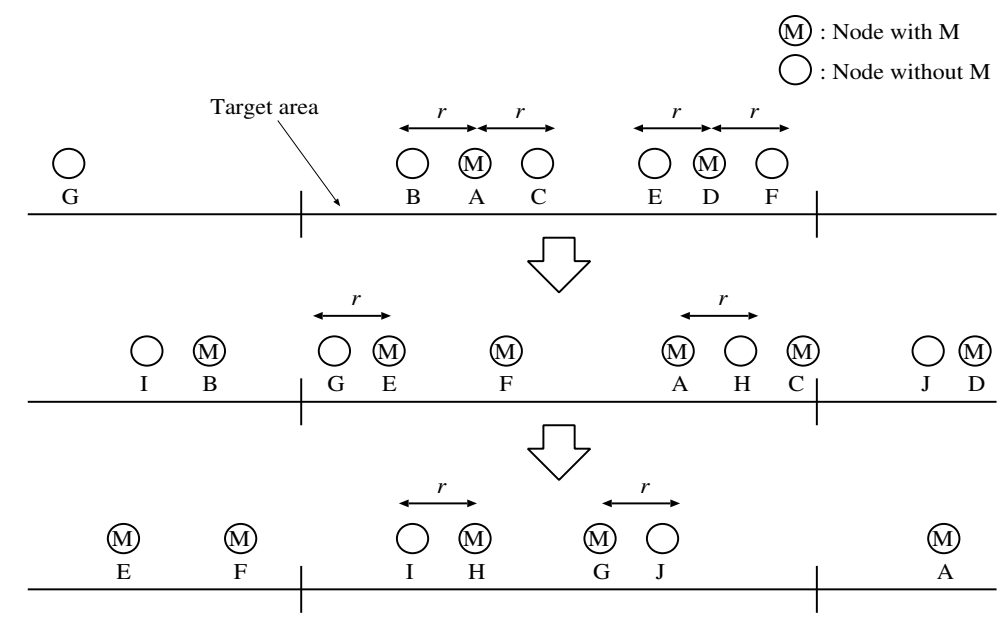

Figure 1: Concept of information floating. Message $M$ is distributed in target area.

in an epidemic manner [6]. The following is the mechanism of epidemic communication in DTNs. Information is carried by mobile nodes and transferred to other nodes when they enter the communication range of each other. As a result of repeating the movement of the nodes carrying information and direct communication among nodes, information is spread widely.

Epidemic communication is applied to information floating (IF), which is a concept that realizes an invisible infrastructure to deliver local information to mobile nodes that enter a target area. IF is realized by repeating information exchanges among mobile nodes by a wireless direct communication function. To eliminate meaningless information diffusion, information exchange is restricted to a designated area. To restrict diffusion, it is often assumed that a mobile node recognizes the designated area and can always get its own position through a GPS devise or techniques that use wireless communication to obtain position information. Figure 1 shows an example of IF. Message $M$ is delivered from nodes A and D to nodes B, C, E, and F. After that, $\mathrm{M}$ is delivered from $\mathrm{E}$ and $\mathrm{A}$ to $\mathrm{G}$ and $\mathrm{H}$ and from $\mathrm{H}$ and $\mathrm{G}$ to $\mathrm{I}$ and $\mathrm{J}$. From such repetition of deliveries, $\mathrm{M}$ is floated in the target area. IF is applicable to such areas as local advertisements, specific communication in disaster situations, and alert systems for pedestrians and vehicles.

The IF concept was first proposed [7] with a detailed algorithm and the simulation results of the proposed method; this proposal was followed by some articles $[8,9,10,11,12,13$, 14, 15]. Since IF is realized by an information exchange between mobile nodes in the target area, the last node with information in the target area might fail to transfer that information to other nodes. In this case, IF ends. In other words, IF's lifetime is limited. In past researches, the IF lifetime is regarded as a main target of research interest or an important metric of performance evaluation.

Previous research [14, 15] provided a simple method to numerically compute the mean lifetime of IF, assuming identical traffic volume and velocity in both the opposite lanes of a road. Introducing such an assumption is reasonable as a first step toward the lifetime analysis because exact analysis of the mean lifetime of IF is difficult even in a one-dimensional 
road, and the previous approximation [14, 15] depends on the simplification realized by the assumption. As seen from this background, the approximation must be extended to explore the effects of other factors on IF. Among them, we focus on the importance of two factors on traffic volumes and derive a new approximation to the mean lifetime of IF assuming different traffic volumes between opposite lanes (Factor 1) and a correlation between traffic volume and velocity (Factor 2). These factors were ignored to simplify analysis in past researches.

Because of Factor 1, we distinguish two types of message transfers in IF. One is the information transfer between two nodes on opposite lanes when they pass each other (typea), and the other is between two nodes in the same lane (type-b). As explained below, the difference in traffic volume between opposite lanes makes it necessary to focus on both type-a and type-b transfers in the analysis of IF lifetimes. Note that in past researches, type-b transfers were ignored in the analysis for simplicity; however, here, we cannot ignore them because of analysis accuracy. Type-b transfers resemble communication through a multi-hop path of wireless multi-hop networks; therefore, the analytical method includes an idea used in the connectivity analysis of mobile multi-hop networks.

Due to Factor 1, the traffic volume differs between opposite lanes. In general, traffic volume and velocity have a correlation (Factor 2). Therefore, the velocity becomes different between opposite lanes due to Factors 1 and 2. To consider this, we use Greenshield's model [16] as a typical model for the correlation between traffic volume and velocity. In this model, the velocity linearly decreases as the node density increases. The difference of velocities between opposite lanes raises a new issue that never appears in the case of identical velocities. We consider this issue to provide an analytical method of the mean lifetime based on Factors 1 and 2.

With this background, we study IF systems on a road with opposite lanes whose traffic volumes are different. We give a new theoretical analysis of the mean lifetime by assuming that two lanes have different traffic volumes and the velocity of each lane is affected by the traffic volume. Although the assumptions were omitted for simplicity in past researches, we reveal how the IF lifetime is affected by the assumptions by introducing the concept of random clumping and utilizing its property. The IF properties are different from MANET and WSN; however, our IF analysis involves the essence of MANET's connectivity analysis, which was not previously included [14, 15].

In Section 2. we explain the model for the mobility of nodes and IF and introduce a concept and some properties of a clump for our analysis in this paper. In Section 3. we theoretically analyze the mean lifetime of IF. In Section 4. we give the numerical results of the mean lifetime of IF and discuss how it is affected by Factors 1 and 2. Section 5 . concludes this paper.

\section{Preliminaries}

\subsection{Distribution and mobility of nodes}

The following are the basic assumptions for the distribution and mobility of nodes:

- Mobile nodes move along a road's opposite lanes, denoted by Lane $_{b}$ and Lane $_{w}$. 


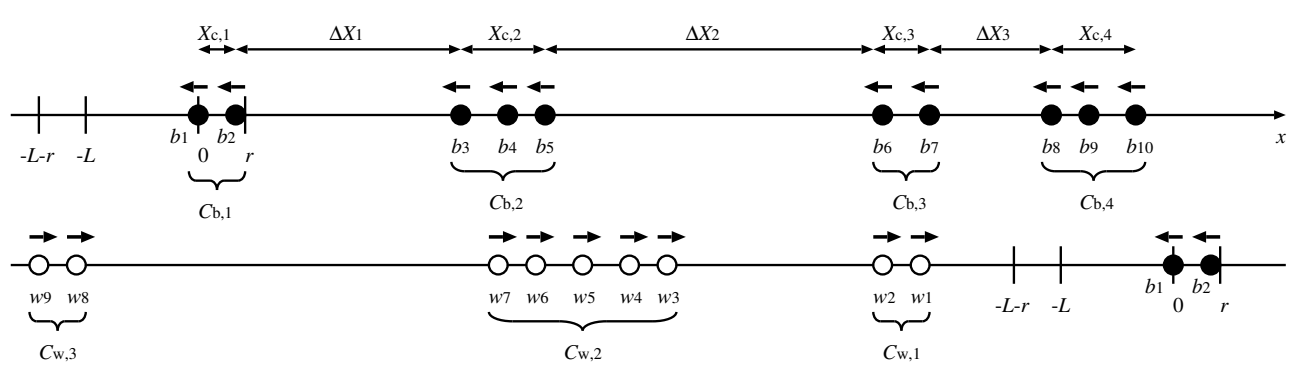

Figure 2: Example of node distribution at $t=t_{0}$ and symbol definitions.

- We draw a line as a road, and nodes on $L_{a n e}$ are black and move left at constant velocity $v_{b}$. Nodes on Lane $_{w}$ are white and move right at constant velocity $v_{w}$. Note that there is no passing in each lane. An example is represented in Fig. 2.

- The spatial distribution of the nodes on $L_{a n e}$ and Lane $_{w}$ obey Poisson distributions of intensities $\lambda_{b}$ and $\lambda_{w}$.

- The traffic volumes of mobile nodes on Lane $_{b}$ and Lane $_{w}$ are denoted by $Q_{b}$ and $Q_{w}$. $Q_{b}=\lambda_{b} v_{b}$ and $Q_{w}=\lambda_{w} v_{w}$.

These assumptions are the same as in other work [14, 15]. In this paper, however, we introduce the following new assumptions, where $Q_{b}, v_{b}$, and $\lambda_{b}$ can be different from $Q_{w}$, $v_{w}$, and $\lambda_{w}$, respectively. To represent a correlation between traffic volume and velocity, we use Greenshield's model [16]:

$$
v_{b}=v_{f}\left(1-\frac{\lambda_{b}}{\lambda_{j}}\right), \quad v_{w}=v_{f}\left(1-\frac{\lambda_{w}}{\lambda_{j}}\right),
$$

where $v_{f}$ is the free velocity and $\lambda_{j}$ is the saturated density of the nodes. Introducing the above assumptions requires a new analytical method for the mean lifetime of IF, as we will explain below.

\subsection{Information floating}

The IF assumptions are almost the same as in previous research [12]. Let $\mathrm{M}$ be a message to be floated. Let $r$ be the communication range of a mobile node. A mobile node knows its own position by such location equipment as GPS. To eliminate meaningless information diffusion, transmission of $\mathrm{M}$ is restricted in interval $[-L, 0]$, where $L$ is a positive real number. Suppose that a node periodically transmits beacon signal. Assume that the beacon signal of a node is received by another node if the distance between these nodes is not longer than $r$. Then, a node can notice that another node enters its communication range based on the beacon signal [9]. If a mobile node with $\mathrm{M}$ is in $[-L, 0]$ and the distance to a neighboring node is not longer than $r$, then the mobile node sends $M$ to the neighboring node because the mobile node receives the beacon signal from the neighboring node. Hence, a sender does not need the position information of the receiver. As a result, the position of each node that 
is receiving $\mathrm{M}$ ranges between $[-L-r, r]$. Therefore, we regard interval $[-L-r, r]$ as the target area.

Let $x(n, t)$ be the coordinate of node $n$ at time $t$. Let $b_{i}$ and $N_{b}$ be a node on Lane $_{b}$ for $i=$ $\cdots,-1,0,1, \cdots$ and the set of nodes on Lane $_{b}$, respectively. Suppose that $x\left(b_{i}, t\right)<x\left(b_{i+1}, t\right)$ for all $i$. In the same manner, for Lane $_{w}$, a node is denoted by $w_{j}$ for $j=\cdots,-1,0,1, \cdots$, and the set of nodes is denoted by $N_{w}$, and $x\left(w_{j+1}, t\right)<x\left(w_{j}, t\right)$ for all $j$. Suppose that $b_{1}$ carries original message $\mathrm{M}$ into $[-L, 0]$ at time $t=t_{0}$ for the first time. This assumption of the origin of IF is slightly different from earlier research [12]. Suppose that $x\left(w_{1}, t_{0}\right) \leq 0$ and $x\left(w_{0}, t_{0}\right)>0$. An example of $t=t_{0}$ is represented in Fig. 2 .

Note that $b_{1}$ can continue IF after $t_{0}$ without nodes $b_{i}$ for $i \leq 0$ because these nodes leave the target area earlier than $b_{1}$ and all the nodes that receive $\mathrm{M}$ from the nodes $b_{i}$ for $i \leq 0$ can receive $\mathrm{M}$ also from $b_{1}$. Nodes $w_{j}$ for $j \leq 0$ never transmit $\mathrm{M}$ because they are on the right of $[-L, 0]$ at $t=t_{0}$ and move right. Hence, we can neglect the nodes $b_{i}$ for $i \leq 0$ and $w_{j}$ for $j \leq 0$ to analyze the lifetime of IF after $t_{0}$.

Define IF's lifetime, which is denoted by $T_{f}$, as the duration time from $t_{0}$ to when all nodes with $\mathrm{M}$ leave interval $[-L-r, r]$ without sending $\mathrm{M}$ to other nodes. In this paper, we theoretically analyze the mean of $T_{f}$, which is denoted by $E\left(T_{f}\right)$.

\subsection{Random clump}

In the following analysis, we utilize some of properties of a clump that consists of small segments randomly distributed on a line. Suppose that the length of a segment is $\alpha$, the starting point of a segment is randomly distributed on a line according to a Poisson process of intensity $\beta$, and a segment extends in the right direction. Some segments overlap and comprise a longer segment than $\alpha$. This longer segment is called a clump. An example of clumps consisting of six and two segments is depicted in Fig. 3. Let $C$ be the length of a clump. Then, from [17], we have

$$
\begin{aligned}
& f_{C}(z \mid C>\alpha)=f_{c}(z, \alpha, \beta) \\
& \quad=\frac{\beta}{e^{\beta \alpha}-1}\left[1+\sum_{i=1}^{\left\lfloor\frac{z}{\alpha}\right\rfloor-1} \frac{(-1)^{i}}{i !}\{\beta(z-(i+1) \alpha)\}^{i-1} e^{-i \beta \alpha}\{\beta(z-(i+1) \alpha)+i\}\right], \\
& \operatorname{Pr}(C>z)=P_{c}(z, \alpha, \beta)=\sum_{i=0}^{\left\lfloor\frac{z}{\alpha}\right\rfloor} \frac{(-1)^{i}}{i !} e^{-i \beta \alpha}\{\beta(z-i \alpha)\}^{i-1}\{\beta(z-i \alpha)+i\}, \\
& E(C)=E_{c}(\alpha, \beta)=\frac{e^{\beta \alpha}-1}{\beta},
\end{aligned}
$$

where $f_{C}(z \mid C>\alpha)$ is the probability density function of $C$ given that $C>\alpha$, and $E(C)$ is the expected value of $C$. 


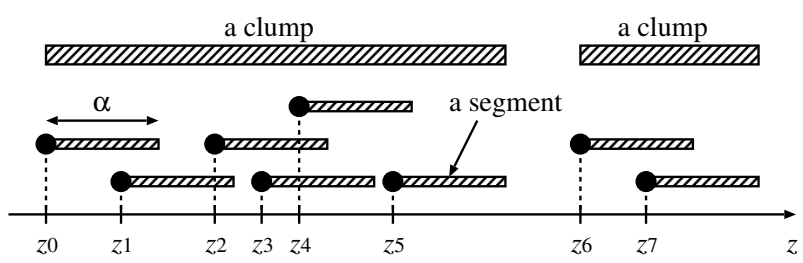

Figure 3: Definition of a clump.

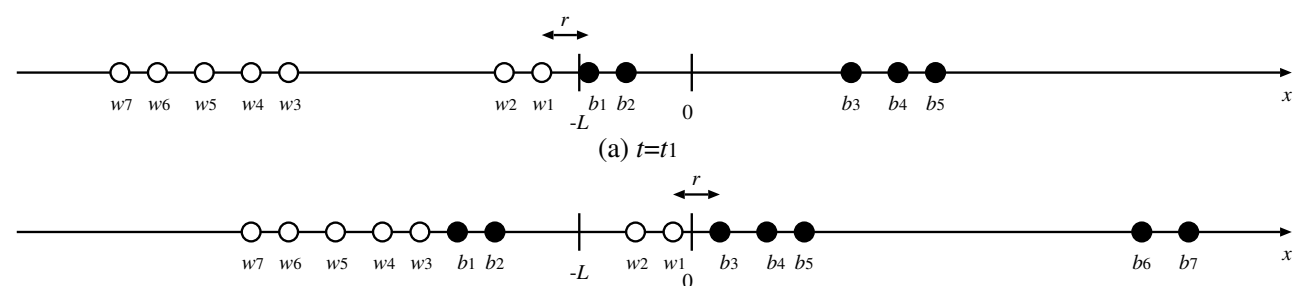

(b) $t=t 2$

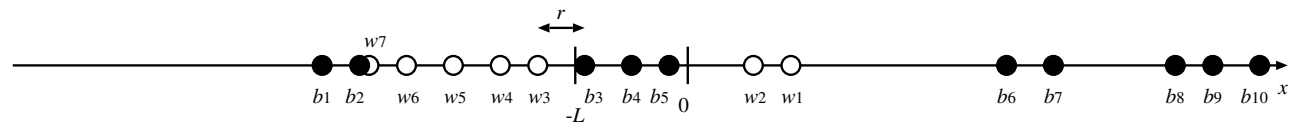

(c) $t=t 3$

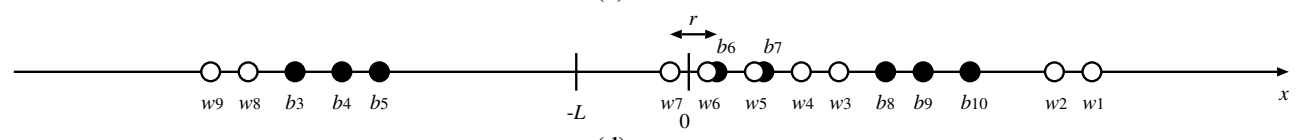

(d) $t=t 4$

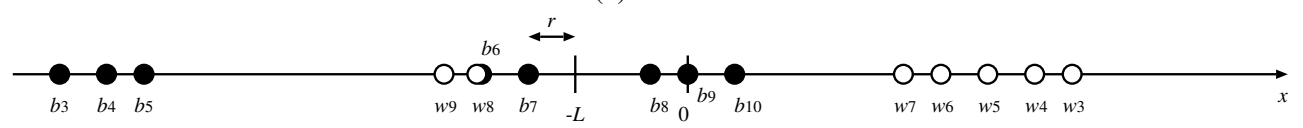

(e) $t=t 5$

Figure 4: Example of IF after $t=t_{0}$, where $v_{b}=v_{w}$. (Situation at $t=t_{0}$ is shown in Fig. 2)

\section{Analysis of mean lifetime of IF}

\subsection{Mechanism of IF}

Next we explain the basic IF mechanism using an example where the velocity is the same. Figures 2 and 4 show an example of IF at $t=t_{0}$ and after $t=t_{0}$. In Fig. 2, $b_{1}$ has original message $\mathrm{M}$ and enters $[-L, 0]$ at $t=t_{0}$. Hence, the floating of $\mathrm{M}$ begins at $t=t_{0}$. From $t=t_{0}$ to $t_{4}, \mathbf{M}$ is delivered from $b_{1}$ to $b_{2}, w_{1}, w_{2}, b_{3}, b_{4}, b_{5}, w_{3}, w_{4}, w_{5}, w_{6}, w_{7}, b_{6}$, and $b_{7}$. Node $b_{7}$ cannot transfer M to any nodes and leaves the target area at $t=t_{5}$. Therefore, IF continues from $t=t_{0}$ to $t_{5}$ and ends at $t=t_{5}$. Hence, $T_{f}=t_{5}-t_{0}$.

This example explains both type-a and type-b deliveries, which are the information deliveries between nodes on opposite lanes and on the same lane. IF continues by repeating these two types of message deliveries and ends if neither type-a nor type-b delivery can deliver $\mathrm{M}$.

In past researches [14, 15], however, type-b delivery is approximately omitted by assuming that a mobile node of $N_{b}$ and $N_{w}$ alternately receives information to continue IF. 
Journal of Advanced Simulation in Science and Engineering

This approximation is expected to work well if the traffic volumes are the same in both lanes because mobile nodes on opposite lanes have identical opportunities to pass each other. On the other hand, a mobile node on a lane has fewer opportunities to pass other nodes if the opposite lane's traffic volume is small.

Note what happens in the case of different traffic volumes between opposite lanes. Consider Figs. 2 and 4 again. From times $t=t_{0}$ to $t_{3}$, message $\mathbf{M}$ is delivered from $b_{1}$ to $w_{1}$, from $w_{1}$ to $b_{3}$, and from $b_{3}$ to $w_{3}$ by type-a delivery. After $t=t_{3}$, only type-b deliveries contribute to keeping IF alive. $\mathrm{M}$ is delivered to $w_{7}$ through $w_{3}, w_{4}, w_{5}$, and $w_{6}$. As seen from this example, such a situation happens if the distance between successive nodes on the same lane is very long and there are dense nodes in the opposite lane. If the balance of the traffic volume is disturbed between opposite lanes, type-b delivery might no longer be negligible.

For the above reasons, in this paper, we focus on type-b delivery to analyze the mean lifetime of IF using a different approach from previous work [14, 15]. A cluster is a set of nodes in the same lane in which the distance between adjacent nodes is not greater than $r$. For example, $\left\{b_{1}, b_{2}\right\},\left\{b_{3}, b_{4}, b_{5}\right\},\left\{w_{1}, w_{2}\right\}$ and $\left\{w_{3}, w_{4}, w_{5}, w_{6}, w_{7}\right\}$ are clusters in Figs. 2 and 4 Let $C_{b, k}$ be the $k$ th cluster of $N_{b}$, and let $C_{w, k}$ be the $k$ th cluster of $N_{w}$. Denote the nodes in $C_{b, k}$ by $b_{H_{k}}, b_{H_{k}+1}, \cdots, b_{T_{k}}$, where $H_{1}=1$ and $H_{k}=T_{k-1}+1$. Let $\Delta X_{k}$ be the distance between $C_{b, k}$ and $C_{b, k+1}$, which means the distance between $b_{T_{k}}$ and $b_{H_{k+1}}$. Let $X_{c, k}$ be the length of $C_{b, k}$, which means the distance between $b_{H_{k}}$ and $b_{T_{k}}$.

We translate successive information exchanges between nodes for the IF as a flow of information along a series of clusters. Consider Figs. 2 and 4, and suppose that the floating of message $\mathrm{M}$ begins with a black node and ends right after a black node leaves the target area. As shown in this example, nodes of $C_{b, 1}$ receive $\mathrm{M}$ through type-b delivery. A node of $C_{w, 1}$ receives $\mathrm{M}$ from that of $C_{b, 1}$, other nodes in $C_{w, 1}$ receive $\mathrm{M}$ through successive type-b deliveries, and a node of $C_{w, 1}$ delivers $\mathrm{M}$ to a node of $C_{b, 2}$. This process is repeated until IF ends.

From the interpretation, $T_{f}$ is represented as

$$
T_{f}=T_{f, 1}=\frac{\sum_{k=1}^{n_{s}} X_{c, k}+\sum_{k=1}^{n_{s}-1} \Delta X_{k}+L+r}{v_{b}},
$$

if the last node holding $\mathrm{M}$ in the target area is a black node in $C_{b, n_{s}}$. Otherwise, the lifetime is extended by the white nodes and

$$
T_{f}=T_{f, 1}+T_{e},
$$

where $T_{e}$ is the extended lifetime and $T_{e}<\frac{\Delta X_{n_{s}}}{v_{b}}$. As the main target of this paper, we analyze $T_{f, 1}$ because $T_{e}$ is relatively small. In (5), $X_{c, k}+r$ is the length of a clump whose starting points of segments are black nodes, and another clump related to the white nodes is examined to evaluate the possibility of successive type-b deliveries in $C_{w, 1}$ in the above example. In this way, $T_{f}$ is deeply related to the clump properties, which appear in our interpretation of the lifetime. In past researches, however, the role of clumps was not considered because type-b deliveries were neglected for approximation. 


\subsection{Analysis of $E\left(T_{f}\right)$}

As mentioned in the preceding section, we focus on the transfers of $\mathrm{M}$ along $C_{b, 1}, C_{b, 2}$, $\ldots$ and compute $T_{f}$ as (5). Although $v_{b}$ can be different from $v_{w}$ due to Factors 1 and 2, we analyze $E\left(T_{f}\right)$ supposing $v_{b} \leq v_{w}$ for the following reason. First, the analysis of $E\left(T_{f}\right)$ becomes difficult if we lose this condition because it is needed for the proofs of some properties in the analysis. Second, we can approximately compute $E\left(T_{f}\right)$ for $v_{b}>v_{w}$ using the formula of $E\left(T_{f}\right)$ for $v_{b} \leq v_{w}$ by changing the colors of the nodes. If we denote the formula of $E\left(T_{f}\right)$ for $v_{b} \leq v_{w}$ by $g_{1}\left(\lambda_{b}, \lambda_{w}, v_{b}, v_{w}\right)$, then we can approximately compute $E\left(T_{f}\right)$ for $v_{b}>v_{w}$ as $g_{1}\left(\lambda_{w}, \lambda_{b}, v_{w}, v_{b}\right)$. Replacing the colors of the nodes slightly changes the situation at $t=t_{0}$ because the color of the node carrying original message $\mathrm{M}$ (i.e., $b_{1}$ ) is also replaced; however, this effect is relatively small, as will be shown in Section 4.3. For these reasons, we analyze $E\left(T_{f}\right)$ supposing that $v_{b} \leq v_{w}$ in the following.

Let $S_{i}$ be an event where $C_{b, i}$ can deliver $\mathrm{M}$ to $C_{b, i+1}$. Considering the probability that $S_{i}$ occurs, $E\left(T_{f}\right)$ is represented as

$$
\begin{aligned}
& E\left(T_{f}\right)=\frac{1}{v_{b}}\left[E\left(X_{c, 1}\right)+L+r+\operatorname{Pr}\left(S_{1}\right)\left\{E\left(\Delta X_{1} \mid S_{1}\right)+E\left(X_{c, 2}\right)\right\}\right. \\
& \quad+\operatorname{Pr}\left(S_{1}, S_{2}\right)\left\{E\left(\Delta X_{2} \mid S_{1}, S_{2}\right)+E\left(X_{c, 3}\right)\right\} \\
& \left.\quad+\operatorname{Pr}\left(S_{1}, S_{2}, S_{3}\right)\left\{E\left(\Delta X_{3} \mid S_{1}, S_{2}, S_{3}\right)+E\left(X_{c, 4}\right)\right\}+\cdots\right] .
\end{aligned}
$$

Because precisely analyzing $\operatorname{Pr}\left(S_{1}, \cdots, S_{i}\right)$ and $E\left(\Delta X_{i} \mid S_{1}, \cdots, S_{i}\right)$ is difficult, we approximately assume that $S_{1}, S_{2}, \cdots$ are independent from each other. In other words, $S_{i}$ and $\Delta X_{i}$ are not affected by $S_{1}, S_{2}, \cdots, S_{i-1}$. From this assumption, we compute

$$
\begin{aligned}
& \operatorname{Pr}\left(S_{1}, \cdots, S_{i}\right)=\operatorname{Pr}\left(S_{1}\right)^{i}, \\
& E\left(\Delta X_{i} \mid S_{1}, \cdots, S_{i}\right)=E\left(\Delta X_{1} \mid S_{1}\right) .
\end{aligned}
$$

Next we substitute Eqs. (8) and (9) into (7):

$$
E\left(T_{f}\right)=\frac{1}{v_{b}}\left\{\frac{E\left(X_{c, 1}\right)+\operatorname{Pr}\left(S_{1}\right) E\left(\Delta X_{1} \mid S_{1}\right)}{1-\operatorname{Pr}\left(S_{1}\right)}+L+r\right\} .
$$

Here, $E\left(X_{c, 1}\right)$ can be computed as $\frac{e^{\lambda_{b} r}-1}{\lambda_{b}}-r$ from $\sqrt{4}$ because $X_{c, k}+r$ corresponds to the length of a clump with segment density $\lambda_{b}$ and segment length $r . \operatorname{Pr}\left(S_{1}\right)$ and $E\left(\Delta X_{1} \mid S_{1}\right)$ can also be rewritten:

$$
\begin{aligned}
& \operatorname{Pr}\left(S_{1}\right)=\int_{-\infty}^{\infty} \operatorname{Pr}\left(S_{1} \mid \Delta X_{1}=x\right) f_{\Delta X_{1}}(x) d x \\
& E\left(\Delta X_{1} \mid S_{1}\right)=\int_{-\infty}^{\infty} x f_{\Delta X_{1}}\left(x \mid S_{1}\right) d x=\int_{-\infty}^{\infty} \frac{x \operatorname{Pr}\left(S_{1} \mid \Delta X_{1}=x\right) f_{\Delta X_{1}}(x)}{\operatorname{Pr}\left(S_{1}\right)} d x,
\end{aligned}
$$

where $f_{\Delta X_{k}}(x)=\lambda_{b} e^{-\lambda_{b}(x-r)}$ for $x>r$ and $f_{\Delta X_{k}}(x)=0$ for $x \leq r$ because $\Delta X_{k}$ obeys an exponential distribution with intensity $\lambda_{b}$ given that it exceeds $r$. Therefore, we only have to analyze $\operatorname{Pr}\left(S_{1} \mid \Delta X_{1}=x\right)$ to compute $E\left(T_{f}\right)$.

To analyze $\operatorname{Pr}\left(S_{1} \mid \Delta X_{1}=x\right)$, we consider the relation between the positions of $C_{b, 1}, C_{b, 2}$, and the nodes of $N_{w}$. Note the example in Fig. 5. A node of $C_{w, k}$ receives $\mathrm{M}$ from a node of 


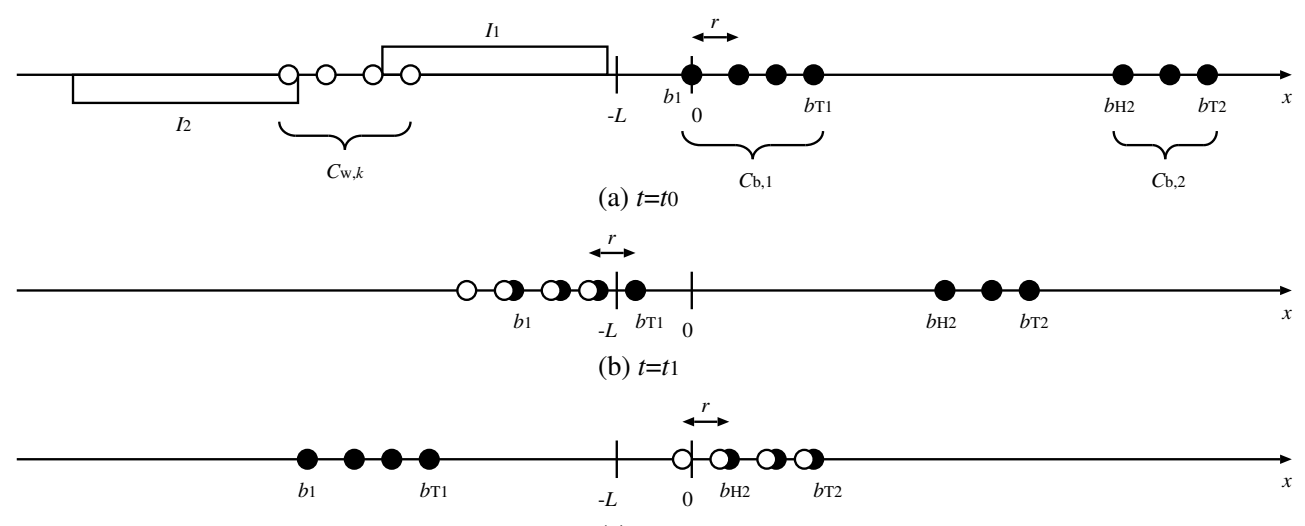

(c) $t=t 2$

Figure 5: Example of $I_{1}$ and $I_{2}$.

$C_{b, 1}$ and distributes $\mathrm{M}$ to the following nodes of $C_{w, k}$, and a node of $C_{w, k}$ having $\mathrm{M}$ sends $\mathrm{M}$ to a node of $C_{b, 2}$. In this manner, $\mathrm{M}$ is delivered from $C_{b, 1}$ to $C_{b, 2}$ by some nodes of $C_{w, k}$. M's delivery from $C_{b, 1}$ to $C_{b, 2}$ is possible if and only if there is at least one node of $N_{w}$ in $I_{1}=\left[x_{1, \ell}, x_{1, r}\right]$ at $t=t_{0}$, there is at least one node of $N_{w}$ in $I_{2}=\left[x_{2, \ell}, x_{2, r}\right]$ at $t=t_{0}$, and the nodes in $I_{1}$ and $I_{2}$ belong to the same cluster of $N_{w}$, where $x_{1, \ell}=-\frac{v_{w}}{v_{b}}\left\{x\left(b_{T_{1}}, t_{0}\right)+L\right\}-L-r$, $x_{1, r}=-\frac{v_{w}}{v_{b}} x\left(b_{T_{1}}, t_{0}\right)+r, x_{2, \ell}=-\frac{v_{w}}{v_{b}}\left\{x\left(b_{H_{2}}, t_{0}\right)+L+r\right\}-L$, and $x_{2, r}=-\frac{v_{w}}{v_{b}}\left\{x\left(b_{H_{2}}, t_{0}\right)-r\right\}$.

To compute $\operatorname{Pr}\left(S_{1} \mid \Delta X_{1}=x\right)$, we consider two cases that have different lengths of the overlap between $I_{1}$ and $I_{2}$. In case (i), the overlap is not smaller than $r$, but in case (ii), it is smaller than $r$. From another viewpoint, in case (i), $\Delta X_{1} \leq\left(\frac{v_{b}}{v_{w}}+1\right) L+r$, and in case (ii), $\Delta X_{1}>\left(\frac{v_{b}}{v_{w}}+1\right) L+r$.

In case (i), there must be a node of $N_{w}$ in the overlap between $I_{1}$ and $I_{2}$ at $t=t_{0}$ if and only if $S_{1}$ occurs (See Appendix A 1.1. for the proof). If $v_{b} \leq v_{w}$, then $\left|I_{1} \cap I_{2}\right|=$ $\frac{v_{w}}{v_{b}}\left(L+r-\Delta X_{1}\right)+L+r$ (See Appendix A 1.2. for the proof). Consequently, we have

$$
\operatorname{Pr}\left(S_{1} \mid \Delta X_{1}=x\right)=1-e^{-\lambda_{w}\left|I_{1} \cap I_{2}\right|}=1-e^{-\lambda_{w}\left\{\frac{v_{w}}{v_{b}}(L+r-x)+L+r\right\}}
$$

because this is the probability that there is at least a node of $N_{w}$ in the overlap between $I_{1}$ and $I_{2}$, and the nodes of $N_{w}$ are distributed based on a Poisson process.

In case (ii), even without a node in the overlap between $I_{1}$ and $I_{2}$ at $t=t_{0}, S_{1}$ can occur if there is a $N_{w}$ cluster whose node is in $I_{1}$ and another node is in $I_{2}$ at $t=t_{0}$, as mentioned above. If $v_{b} \leq v_{w}$, then such a cluster exists if and only if the length of a clump whose starting points of segments are coordinate $x=x_{2, r}-r$ and the nodes of $N_{w}$ at the right side of $x=x_{2, r}-r$ is not smaller than $\frac{v_{w}}{v_{b}}\left(\Delta X_{1}-L-r\right)-L+r$ (See Appendix A.1.3. for the proof). Consequently, from (3) we have

$$
\operatorname{Pr}\left(S_{1} \mid \Delta X_{1}=x\right)=P_{c}\left(\frac{v_{w}}{v_{b}}(x-L-r)-L+r, r, \lambda_{w}\right) .
$$

Finally, we can compute $E\left(T_{f}\right)$ by substituting (13) and (14) into (10), (11), and (12). 


\subsection{Closed form of $E\left(T_{f}\right)$ for a special case}

In the above subsections, we successfully analyzed $E\left(T_{f}\right)$ for a case of unbalanced traffic volume of opposite lanes and correlation between the traffic volume and velocity of the nodes by a new theoretical method that takes the factor of type-b delivery into account. Although our formula is slightly complicated because it includes an integral term, it can also be applied to a special case that includes the previous case [14, 15] (i.e., $Q_{b}=Q_{w}$, $v_{b}=v_{w}$, and $\lambda_{b}=\lambda_{w}$ ), and the formula becomes a simple closed form for such a special case as follows. By substituting condition $v_{b}=v_{w}$ into (11) and (12), we have

$$
\begin{aligned}
& \operatorname{Pr}\left(S_{1}\right)=1+\frac{\lambda_{b}\left\{e^{-2 \lambda_{b} L-\lambda_{w} r}-e^{-\lambda_{w}(2 L+r)}\right\}}{\lambda_{b}-\lambda_{w}}-\frac{\lambda e^{-\lambda_{b}(2 L-r)}}{\lambda_{b} e^{\lambda r}+\lambda_{w}}, \\
& E\left(\Delta X_{1} \mid S_{1}\right) \\
& =\frac{1}{\operatorname{Pr}\left(S_{1}\right)}\left[\frac{1}{\lambda_{b}}+r+\frac{\lambda_{b} e^{-\lambda_{w} r}}{\Delta \lambda^{2}}\left[e^{-2 \lambda_{b} L}\{1+\Delta \lambda(2 L+r)\}-e^{-2 \lambda_{w} L}(1+\Delta \lambda r)\right]\right. \\
& -\frac{e^{-\lambda_{b}(2 L-r)}}{\lambda_{b}\left(\lambda_{b} e^{\lambda r}+\lambda_{w}\right)^{2}}\left[\lambda_{w}\left(\lambda_{w}+2 \lambda_{b} \lambda L\right)+\lambda_{b} e^{\lambda r}\left\{\lambda_{b}+2 \lambda_{w}+\lambda_{b}(2 L+r) \lambda\right\}\right],
\end{aligned}
$$

where $\Delta \lambda=\lambda_{b}-\lambda_{w}$. From these equations and (10), we obtain a simple closed form of $E\left(T_{f}\right)$.

\section{Numerical results and discussions}

In the following, we obtain the numerical and simulation results of $E\left(T_{f}\right)$. We consider warning of an accident on a road [14, 15] and advertisement of a shop on a road as applications of IF. As in [14, 15], we assume that mobile nodes are vehicles moving along the road and that direct communication between nodes are realized by ad hoc mode of wireless LAN. Then, $r$ can take various value as in [14, 15].

In Sections 4.1. and 4.2., we set $v_{b}$ and $v_{w}$ a constant value, and in Section 4.3. $v_{b}$ and $v_{w}$ are computed by (1) to observe effects of Factor 2. Based on the above assumptions, we set $v_{b}$ and $v_{w}$ to $15 \mathrm{~m} / \mathrm{sec}=54 \mathrm{~km} /$ hour. In Section 4.3. this value is used as the maximum value of (1).

In the simulation, we deploy nodes that move left and right based on a Poisson process with intensities $\lambda_{b}$ and $\lambda_{w}$ on the x-axis. According to the assumption in Section 2.1., we move the nodes, execute the information transmissions, and calculate the time when all nodes with $\mathrm{M}$ leave $[-L-r, r]$. We repeat these procedures 10,000 times and calculate $E\left(T_{f}\right)$ as the mean value of these times.

\subsection{Basic tendency of $E\left(T_{f}\right)$}

Figure 6 shows the numerical results of $E\left(T_{f}\right)$ with the simulation results. In Figs.6(a), (b), and (c), the horizontal axis represents $L, r$, and $Q$, respectively. To show the basic tendencies of $E\left(T_{f}\right)$, we set $v_{b}$ and $v_{w}$ to a constant value: $15 \mathrm{~m} / \mathrm{sec}$. We also set $Q_{b}=Q_{w}=\frac{Q}{2}$, which means that the traffic volumes of opposite lanes are the same. From these relations, $\lambda_{b}$ and 
$\lambda_{w}$ are computed as $\frac{Q_{b}}{v_{b}}=\frac{Q_{w}}{v_{w}}=\frac{Q}{30}$. In Fig. 6 (a), $L$ varies from $10 \mathrm{~m}$ to $400 \mathrm{~m}$ while $r=50$ m. In Fig. 6(b), $r$ varies from $10 \mathrm{~m}$ to $350 \mathrm{~m}$ while $L=50 \mathrm{~m}$. In Fig. 6(c), $Q$ varies from $0.075 \mathrm{sec}^{-1}$ to $1.4 \mathrm{sec}^{-1}$ while $r=50 \mathrm{~m}$.

From Fig. 6, we can confirm that the numerical results agree well with the simulation results, which means that our formula works well for a special case where $Q_{b}=Q_{w}, v_{b}=v_{w}$, and $\lambda_{b}=\lambda_{w}$. We can also see that $E\left(T_{f}\right)$ rapidly increases with each $L, r$, and $Q$. This indicates that it is important to use appropriate values of $r$ and $L$ for a given $Q$ to realize the desired value of $E\left(T_{f}\right)$.

The desired value of $E\left(T_{f}\right)$ depends on the application of IF. If IF is used for warning of an accident, IF should continue until the accident handling finishes. In this case, the desired value of the lifetime may be some hours. For a long term advertisement, the desired value of the lifetime of IF may be all the day. Hence, we have to decide the desired value of $E\left(T_{f}\right)$ suitable to the application, and have to decide the values of other parameters to satisfy the desired value. For example, if the desired value is 1 hour in Fig. 6 (a), we have to set $L$ larger than $180 \mathrm{~m}$ for $Q=0.6 \mathrm{sec}^{-1}$ and $r=50 \mathrm{~m}$.

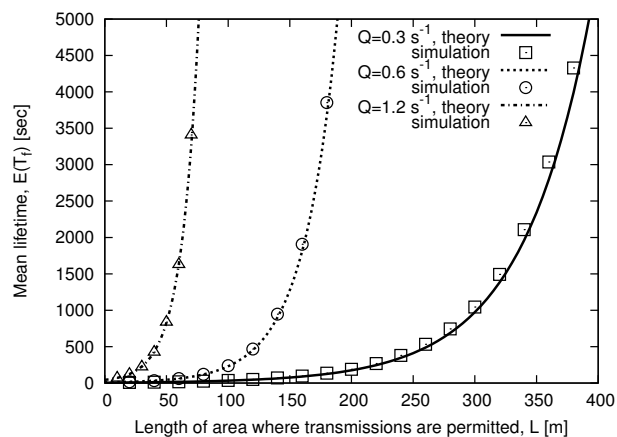

(a) Effect of $L$, where $r=50 \mathrm{~m}$.

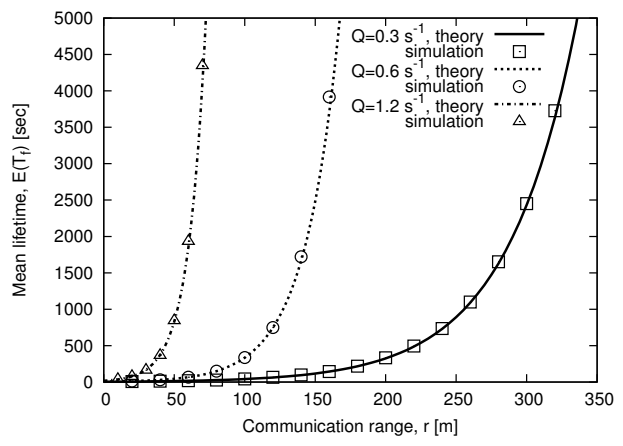

(b) Effect of $r$, where $L=50 \mathrm{~m}$.

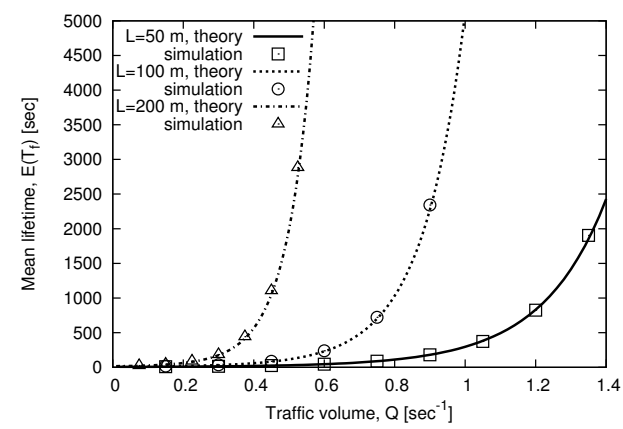

(c) Effect of $Q$, where $r=50 \mathrm{~m}$.

Figure 6: Results of $E\left(T_{f}\right)$, where $Q_{b}=Q_{w}=\frac{Q}{2}$ and $v_{b}=v_{w}=15 \mathrm{~m} / \mathrm{sec}$. 


\subsection{Effect of different traffic volumes between opposite lanes: Factor 1}

Figure 7 shows the effect of the unevenness between traffic volumes of opposite lanes (Factor 1) on $E\left(T_{f}\right)$. The horizontal axis represents $\frac{Q_{b}}{Q}$, which denotes the ratio of the traffic volume of one lane to the total traffic volume. $\frac{Q_{b}}{Q}=0.5$ means that the traffic volumes of opposite lanes are the same, and the difference between them increases if $\frac{Q_{b}}{Q}$ approaches 0 or 1. Figure 7 (a) shows the results for $L \ll r(L=50 \mathrm{~m}$ and $r=200 \mathrm{~m})$, and Fig. 7(b) shows them for $L \gg r(L=200 \mathrm{~m}$ and $r=50 \mathrm{~m})$. In these figures, $v_{b}=v_{w}=15 \mathrm{~m} / \mathrm{sec}$.

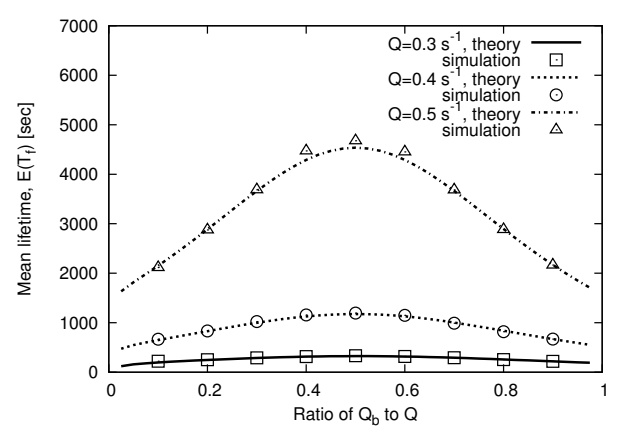

(a) $L=50 \mathrm{~m}$ and $r=200 \mathrm{~m}$.

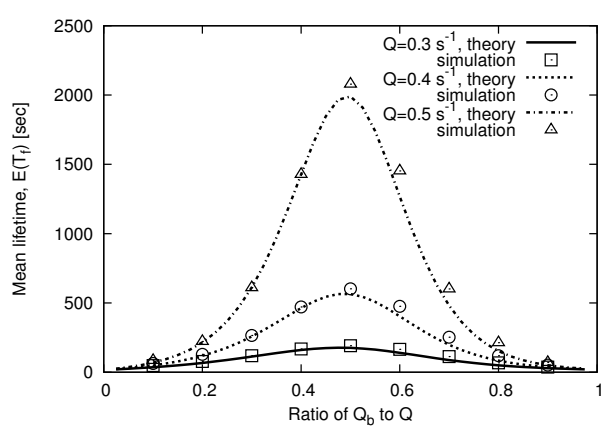

(b) $L=200 \mathrm{~m}$ and $r=50 \mathrm{~m}$.

Figure 7: Relation between $E\left(T_{f}\right)$ and $\frac{Q_{b}}{Q}$, where $v_{b}=v_{w}=15 \mathrm{~m} / \mathrm{sec}$.

From Fig. 7, we can confirm that the numerical results agree well with the simulation results, which means that our formula successfully computed $E\left(T_{f}\right)$ capturing the characteristic of Factor 1. Figures 7 (a) and (b) also confirm that the unevenness between the traffic volumes of opposite lanes greatly reduces $E\left(T_{f}\right)$, especially if $L \gg r$, for the following reason. In general, the effect of type-a delivery is weakened if the unevenness between traffic volumes increases for both $L \ll r$ and $L \gg r$ cases. For $L \ll r$, however, $E\left(T_{f}\right)$ does not greatly decrease even if the unevenness increases because type-b delivery compensates the degradation of type-a delivery because $r$ is large. On the other hand, in the case of $L \gg r$, because the effect of type-b delivery is also weakened, $E\left(T_{f}\right)$ greatly decreases when the unevenness is large. These results indicate that we must carefully choose such parameters as $r$ and $L$ especially if there is a big difference between the traffic volumes of opposite lanes.

\subsection{Effect of correlation between traffic volume and velocity: Factor 2}

As mentioned, traffic volume and velocity have a correlation. To represent it, we use Greenshield's model, where the velocity for each lane is represented by (1). Figure 8 shows an example of the relation among $v_{b}, v_{w}$, and $\lambda$, where $v_{f}=15 \mathrm{~m} / \mathrm{sec}$ and $\lambda_{j}=0.12 \mathrm{~m}^{-1}$. Figure 8 shows that a large unevenness of traffic volumes causes a big difference between the velocities in opposite lanes. $v_{b} \ll v_{w}$ if $\lambda_{b} \gg \lambda_{w}$, and $v_{b} \gg v_{w}$ if $\lambda_{b} \ll \lambda_{w}$.

Figure 9 shows the $E\left(T_{f}\right)$ results considering the correlation between traffic volume and velocity (Factor 2), where $v_{f}=15 \mathrm{~m} / \mathrm{sec}, \lambda_{j}=0.12 \mathrm{~m}^{-1}$, and $r=50 \mathrm{~m} . \lambda=0.06 \mathrm{~m}^{-1}$ in 


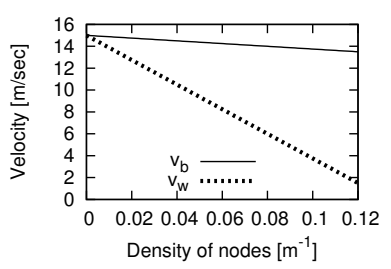

(a) $\lambda_{b}=0.1 \lambda$ and $\lambda_{w}=0.9 \lambda$.

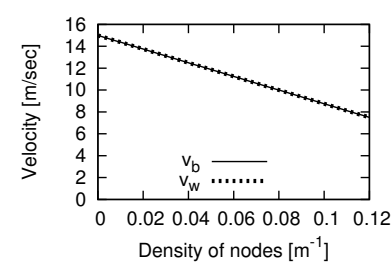

(b) $\lambda_{b}=0.5 \lambda$ and $\lambda_{w}=0.5 \lambda$.

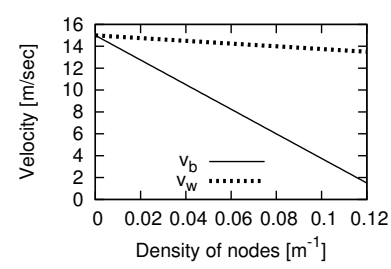

(c) $\lambda_{b}=0.9 \lambda$ and $\lambda_{w}=0.1 \lambda$.

Figure 8: Velocities of nodes, where $v_{f}=15 \mathrm{~m} / \mathrm{sec}$ and $\lambda_{j}=0.12 \mathrm{~m}^{-1}$.

Figs. 9 (a) and (b) and $\lambda=0.11 \mathrm{~m}^{-1}$ in Figs. 9 (c) and (d). $\lambda_{b}=\lambda_{w}\left(\frac{\lambda_{b}}{\lambda}=0.5\right)$ in Figs. 9 (a) and (c) and $\lambda_{b} \gg \lambda_{w}\left(\frac{\lambda_{b}}{\lambda}=0.9\right)$ in Figs. 9 (b) and (d). This figure also shows the $E\left(T_{f}\right)$ results without correlation (i.e., $v_{b}=v_{w}=15 \mathrm{~m} / \mathrm{sec}$ ) for comparison. The horizontal axis represents $L$.

From Fig. 9, the correlation between traffic volume and velocity greatly increases $E\left(T_{f}\right)$ if $\frac{\lambda_{b}}{\lambda}=0.9$ (Figs. 9 (b) and (d)), while the correlation only slightly affects $E\left(T_{f}\right)$ if $\frac{\lambda_{b}}{\lambda}=0.5$ (Figs. 9 (a) and (c)). Especially for a large $\lambda$ and great unevenness between traffic volumes and velocities in opposite lanes (Fig. 9 (d)), the effect of correlation is quite large, and $E\left(T_{f}\right)$ in this case becomes larger than that for a case of equal traffic volumes and velocities (Fig. 9 (c)). One reason why Factor 2 increases $E\left(T_{f}\right)$ is that the velocities of the nodes decrease on average due to Factor 2. For reference, Figs. 9 (b) and (d) also show the $E\left(T_{f}\right)$ results, assuming that the velocities of all the nodes are the same and equal to $\min \left\{v_{b}, v_{w}\right\}$. From these results, $E\left(T_{f}\right)$ with the correlation is larger than that assuming the same and slow velocity. This means that not only decrease of the velocities on average but also the characteristics of Factor 2, where the velocity is more degraded for denser lanes, contributes to the $E\left(T_{f}\right)$ increase.

Finally, we show that we can use the $E\left(T_{f}\right)$ formula derived in the preceding section, which supposed that $v_{b} \leq v_{w}$, for a case of $v_{b}>v_{w}$. Figure 10 shows the numerical results of $E\left(T_{f}\right)$ considering Factors 1 and 2 for $\frac{\lambda_{b}}{\lambda}=0.7$ and 0.9 (here $v_{b} \leq v_{w}$ ) with the simulation results of $E\left(T_{f}\right)$ for $\frac{\lambda_{b}}{\lambda}=0.1,0.3,0.7$, and 0.9. These figures confirm that the simulation results of $E\left(T_{f}\right)$ for $\frac{\lambda_{b}}{\lambda}=0.3$ and 0.7 are almost the same, and the numerical results of $E\left(T_{f}\right)$ of $\frac{\lambda_{b}}{\lambda}=0.7$ agree well with both of them. In the same manner, the numerical results of $E\left(T_{f}\right)$ of $\frac{\lambda_{b}}{\lambda}=0.9$ agree well with both the simulation results for $\frac{\lambda_{b}}{\lambda}=0.1$ and 0.9 . Hence, we can approximately compute $E\left(T_{f}\right)$ for $v_{b}>v_{w}$ using the formula derived in the preceding section and changing the colors of the nodes.

\section{Conclusions}

In this paper, we considered information floating (IF), in which information seems to float on a specific area by wireless direct communications between mobile nodes, and theoretically analyzed its mean lifetime in a one-dimensional road network. Our analysis addressed the effects of the difference between traffic volumes in opposite lanes (Factor 1) and the 


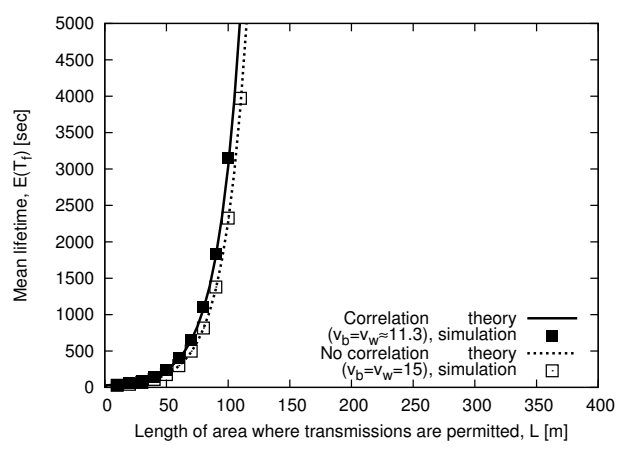

(a) $\lambda=0.06 \mathrm{~m}^{-1}$ and $\frac{\lambda_{b}}{\lambda}=0.5$.

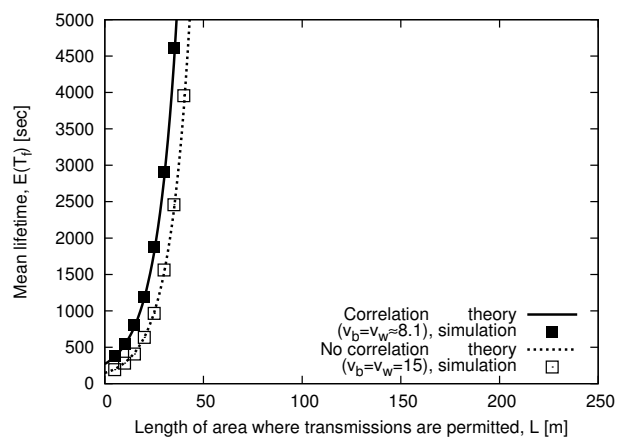

(c) $\lambda=0.11 \mathrm{~m}^{-1}$ and $\frac{\lambda_{b}}{\lambda}=0.5$.

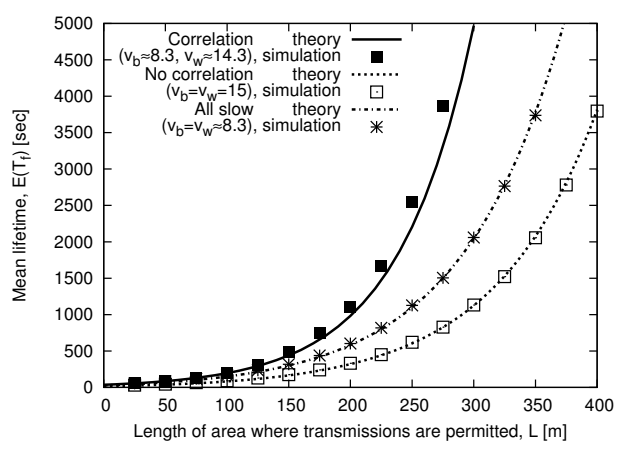

(b) $\lambda=0.06 \mathrm{~m}^{-1}$ and $\frac{\lambda_{b}}{\lambda}=0.9$.

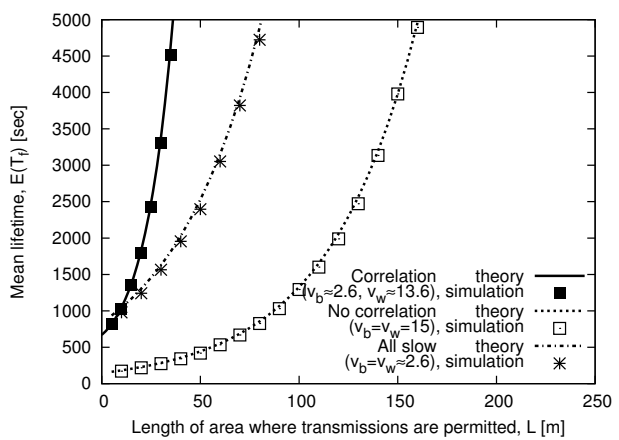

(d) $\lambda=0.11 \mathrm{~m}^{-1}$ and $\frac{\lambda_{b}}{\lambda}=0.9$.

Figure 9: Relation between $E\left(T_{f}\right)$ and $L$, where $v_{b}$ and $v_{w}$ are given by (1).

correlation between traffic volume and velocity of the vehicles in each lane (Factor 2); both factors were ignored in past researches. Our numerical results showed that both of the above factors greatly affect the mean lifetime of IF: i) Even if the total traffic volume of the opposite lanes is the same, the mean lifetime significantly decreases if the unevenness of the traffic volumes becomes large; ii) The correlation between traffic volume and velocity significantly increases the mean lifetime longer even in a case of unbalanced traffic volumes between opposite lanes.

In this paper, we considered Factors 1 and 2 as natural extensions of the analysis of IF in a simple one-dimensional road model. Although we considered a two-lane road in this paper, we should consider IF on a road with more lanes. Also, transient characteristics are important. These issues should be considered in future works. Future works also include analysis of the mean IF lifetime in a more complicated mobility model such as a two-dimensional road model.

\section{Acknowledgments}

This work is partially supported by JSPS KAKENHI Grant Number 25420360. The authors thank Shunsuke Hayatsu and Yuta Mifune of the Graduate School of Science and Technol- 


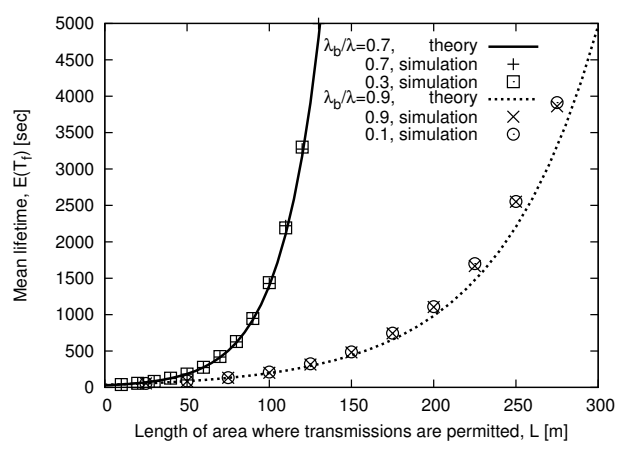

(a) $\lambda=0.06 \mathrm{~m}^{-1}$.

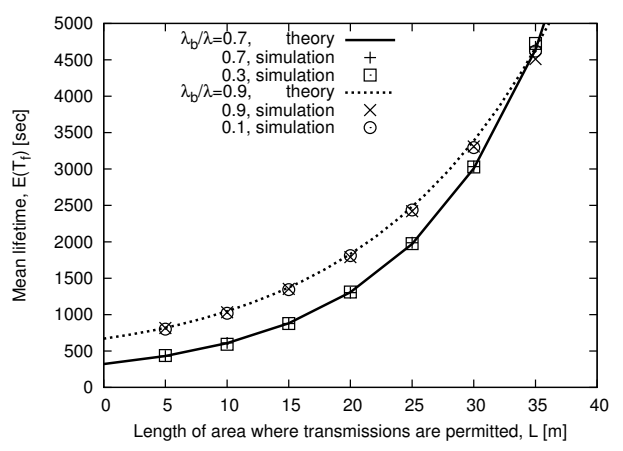

(b) $\lambda=0.11 \mathrm{~m}^{-1}$.

Figure 10: Comparison between $E\left(T_{f}\right)$ for cases $v_{b} \leq v_{w}$ and $v_{b}>v_{w}$.

ogy, Niigata University for their help. The authors thank anonymous reviewers for their valuable comments.

\section{Appendix A. Proofs of properties in Section 3.2.}

\section{1.}

In this appendix, we prove that in case (i) (i.e., $\left|I_{1} \cap I_{2}\right| \geq r$ ), a white node must exist in the overlap between $I_{1}$ and $I_{2}$ at $t=t_{0}$ if and only if $S_{1}$ occurs.

First, suppose that there is a white node in the overlap between $I_{1}$ and $I_{2}$ at $t=t_{0}$. In this case, $S_{1}$ obviously occurs because this node can deliver M from $C_{b, 1}$ to $C_{b, 2}$. Next, suppose that there is no white node in the overlap between $I_{1}$ and $I_{2}$ at $t=t_{0}$. In this case, even if there are white nodes in both $I_{1}$ and $I_{2}$ at $t=t_{0}$, it is impossible that these nodes belong to the same cluster because there is a gap longer than $r$ (i.e., $I_{1} \cap I_{2}$ ) between them. Therefore, $S_{1}$ never occurs in this case. From these facts, this property holds.

\section{2.}

In this appendix, we prove that if $v_{b} \leq v_{w}$ and $I_{1} \cap I_{2} \neq \emptyset$, then $\left|I_{1} \cap I_{2}\right|=\frac{v_{w}}{v_{b}}\left(L+r-\Delta X_{1}\right)+L+r$.

If $I_{1} \cap I_{2} \neq \emptyset$, then $\left|I_{1} \cap I_{2}\right|$ can obviously be represented as $\min \left\{x_{1, r}, x_{2, r}\right\}-\max \left\{x_{1, \ell}, x_{2, \ell}\right\}$ because $I_{1}=\left[x_{1, \ell}, x_{1, r}\right]$ and $I_{2}=\left[x_{2, \ell}, x_{2, r}\right]$ are intervals. Here, from the definitions of $b_{T_{1}}$ and $b_{H_{2}}$, we generally have $x\left(b_{H_{2}}, t_{0}\right)>x\left(b_{T_{1}}, t_{0}\right)+r$. From this inequality and the definitions of $x_{1, r}, x_{1, \ell}, x_{2, r}$, and $x_{2, \ell}$, if $v_{b} \leq v_{w}$, then we have $x_{2, r}<x_{1, r}$ and $x_{2, \ell}<x_{1, \ell}$. From these inequalities and the above equation of $\left|I_{1} \cap I_{2}\right|$, we have $\left|I_{1} \cap I_{2}\right|=x_{2, r}-x_{1, \ell}$. From this equation and the definitions of $x_{2, r}$ and $x_{1, \ell}$, we have $\left|I_{1} \cap I_{2}\right|=\frac{v_{w}}{v_{b}}\left(L+r-\Delta X_{1}\right)+L+r$.

\section{3.}

In this appendix, we prove that in case (ii) (i.e., $\left|I_{1} \cap I_{2}\right|<r$ ), if $v_{b} \leq v_{w}$, then there is a cluster of $N_{w}$ whose node is in $I_{1}$ and another node is in $I_{2}$ at $t=t_{0}$ if and only if the length 


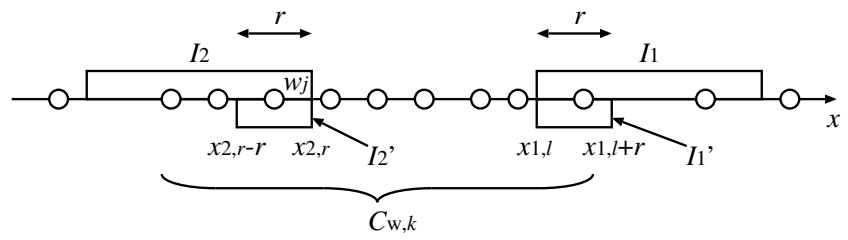

(a) Situation where $S_{1}$ occurs in case (ii)

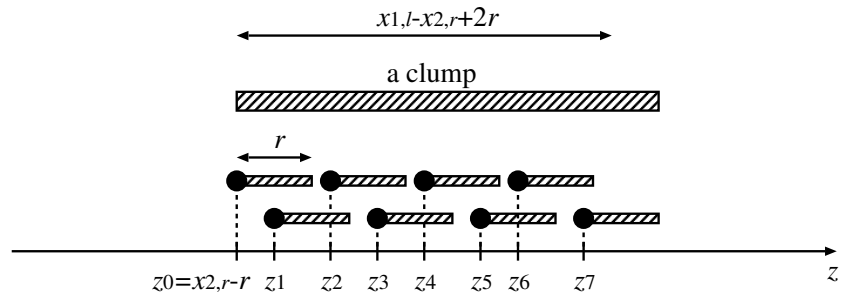

(b) Situation identical to (a)

Figure 11: Example of $I_{1}^{\prime}$ and $I_{2}^{\prime}$.

of a clump whose starting points of segments are coordinate $x=x_{2, r}-r$ and the nodes of $N_{w}$ at the right side of $x=x_{2, r}-r$ is not smaller than $\frac{v_{w}}{v_{b}}\left(\Delta X_{1}-L-r\right)-L+r$.

If $v_{b} \leq v_{w}$, then we have $\left|I_{1}\right| \geq r$ and $\left|I_{2}\right| \geq r$ from the definitions of $x_{1, r}, x_{1, \ell}, x_{2, r}$, and $x_{2, \ell}$. Here, we consider the left part of $I_{1}$ of length $r$ and the right part of $I_{2}$ of length $r$, denoted by $I_{1}^{\prime}$ and $I_{2}^{\prime}$, respectively, (Fig. 11). $I_{1}^{\prime}=\left[x_{1, \ell}, x_{1, \ell}+r\right]$ and $I_{2}^{\prime}=\left[x_{2, r}-r, x_{2, r}\right]$. Obviously, the event where there is a $N_{w}$ cluster whose node is in $I_{1}$ and another node in $I_{2}$ is identical to the event where there is a $N_{w}$ cluster whose node is in $I_{1}^{\prime}$ and another node is in $I_{2}^{\prime}$. The latter event occurs if and only if there is a white node in $I_{2}^{\prime}$ (denoted by $w_{j}$ ), and the rightmost node of the cluster including $w_{j}$ is at the right side of $x=\min I_{1}^{\prime}=x_{1, \ell}$. This event is identical to one where the length of a clump whose starting points of segments are coordinate $x=x_{2, r}-r$ and the nodes of $N_{w}$ at the right side of $x=x_{2, r}-r$ is not smaller than $x_{1, \ell}-x_{2, r}+2 r$. Here, $x_{1, \ell}-x_{2, r}+2 r$ can be computed as $\frac{v_{w}}{v_{b}}\left(\Delta X_{1}-L-r\right)-L+r$ from the definitions of $x_{1, \ell}$ and $x_{2, r}$. Therefore, this property holds.

\section{References}

[1] C. E. Perkins: Ad Hoc Networking, Addison-Wesley, Boston, 2008.

[2] P. Santi: Topology control in wireless ad hoc and sensor networks, ACM Computing Surveys, 37:2 (2005), 164-194.

[3] I. F. Akyildiz, X. Wang, W. Wang: Wireless mesh networks: a survey, Computer Networks, 47:4 (2005), 445-487.

[4] K. Fall: A delay-tolerant network architecture for challenged internets, in Proc. 2003 conference on Applications, technologies, architectures, and protocols for computer communications, Karlsruhe, 2003, 27-34. 
Journal of Advanced Simulation in Science and Engineering

[5] F. Warthman: Delay tolerant networks (DTNs), A tutorial, DTN Research Group, Internet Draft, 2003.

[6] A. Vahdat and D. Becker: Epidemic routing for partially connected ad hoc networks, Technical Report, Duke University, 2000.

[7] A.V. Castro, G.D.M. Serugendo, D. Konstantas: Hovering Information - SelfOrganising Information that Finds its Own Storage, Technical Report, School of Computer Science and Information Systems, Birkbeck College, 2007.

[8] E. Hyytia, J. Virtamo, P. Lassila, J. Kangasharju and J. Ott: When Does Content Float? Characterizing Availability of Anchored Information in Opportunistic Content Sharing, in Proc. INFOCOM IEEE, Shanghai, 2011, 3137-3145.

[9] J. Ott, E. Hyytia, P. Lassila, J. Kangasharju and S. Santra: Floating Content for Probabilistic Information Sharing, Pervasive and Mobile Computing, 7:6 (2011), 671-689.

[10] E. Hyytia, P. Lassila, J. Ott and J. Kangasharju: Floating information with stationary nodes, in Proc. Eighth Workshop on Spatial Stochastic Models for Wireless Networks (SpaSWin), Paderborn, 2012, 361-366.

[11] M. S. Desta, E. Hyytia, J. Ott and J. Kangasharju: Characterizing Content Sharing Properties for Mobile Users in Open City Squares, in Proc. 10th Annual IEEE/IFIP Conference on Wireless On-Demand Network Systems and Services (WONS), Banff, 2013, 147-154.

[12] J. Virtamo, E. Hyytiä, P. Lassila: Criticality condition for information floating with random walk of nodes, Performance Evaluation, 70:2 (2013), 114-123.

[13] M. Ciocan, C. Dobre, C.X. Mavromoustakis, G. Mastorakis: Analysis of Vehicular Storage and Dissemination Services based on Floating Content, in Proc. of International Workshop on Enhanced Living EnvironMENTs (ELEMENT 2014), 6th International Conference on Mobile Networks and Management (MONAMI 2014), Wuerzburg, 2014, 387-400.

[14] B. Liu, B. Khorashadi, D. Ghosal, C. N. Chuah, H.M. Zhang: Analysis of the information storage capability of VANET for highway and city traffic, Transportation Research Part C: Emerging Technologies, 23 (2012), 68-84.

[15] B. Xie, Y. W. Chen, M. Xu, and Y. G. Wang: Mathematical Modeling of Locally Information Storage Capability of VANET for Highway Traffic, Applied Mechanics and Materials, 373-375 (2013), 1914-1919.

[16] F. L. Mannering, S. S. Washburn, and W. P. Kilareski: Principles of highway engineering and traffic analysis, Second Edition, John Wiley \& Sons, New Jersey, 1998.

[17] P. Hall: Introduction to the theory of coverage processes, John Wiley \& Sons, New York, 1988. 